hep-th/0205316

SLAC-PUB-9228

HUTP-02/A019

\title{
de Sitter Space in Non-Critical String Theory
}

\author{
Alexander Maloney ${ }^{\dagger}$, Eva Silverstein* and Andrew Strominger ${ }^{\dagger}$
}

\begin{abstract}
Supercritical string theories in $D>10$ dimensions with no moduli are described, generalizing the asymmetric orientifold construction of one of the authors [ [ 1 . By taking the number of dimensions to be large and turning on fluxes, dilaton potentials are generated with nontrivial minima at arbitrarily small cosmological constant and D-dimensional string coupling, separated by a barrier from a flat-space linear dilaton region, but possibly suffering from strong coupling problems. The general issue of the decay of a de Sitter vacuum to flat space is discussed. For relatively small barriers, such decays are described by gravitational instantons. It is shown that for a sufficiently large potential barrier, the bubble wall crosses the horizon. At the same time the instanton decay time exceeds the Poincare recurrence time. It is argued that the inclusion of such instantons is neither physically meaningful nor consistent with basic principles such as causality. This raises the possibility that such de Sitter vacua are effectively stable. In the case of the supercritical flux models, decays to the linear dilaton region can be forbidden by such large barriers, but decays to lower flux vacua including AdS minima nevertheless proceed consistently with this criterion. These models provide concrete examples in which cosmological constant reduction by flux relaxation can be explored.
\end{abstract}

$\dagger$ Department of Physics, Harvard University, Cambridge, MA 02138

* SLAC and Department of Physics, Stanford University, Stanford, CA 94309

To appear in Proceedings of Stephen Hawking's 60th Birthday Symposium

Invited talk presented at Stephen Hawking 60th Birthday Celebration Workshop and Conference, The Future of Theoretical Physics and Cosmology, DAMPT, Cambridge, UK $1 / 7 / 2002-1 / 11 / 2002$

Work supported in part by the Department of Energy contract DE-AC03-76SF00515. 


\section{Contents}

1. Introduction . . . . . . . . . . . . . . . . . . . . . . . . . . . . . . . . . 1

2. de Sitter Compactifications of Super-Critical String Theory . . . . . . . . . . . . . 3

2.1. Asymmetric Orientifolds in Non-Critical String Theory . . . . . . . . . . . . . 1

2.2. de Sitter Solutions . . . . . . . . . . . . . . . . . . . . . . . . . 9

2.3. Solutions With Small $\Lambda$. . . . . . . . . . . . . . . . . . . . . . . . . . . 10

3. Metastability of the de Sitter Vacuum . . . . . . . . . . . . . . . . . . . . . . 11

3.1. The Instantons . . . . . . . . . . . . . . . . . . . . . . . . . . . . . . . 12

3.2. Causality . . . . . . . . . . . . . . . . . . . . . . . . . . . . . . . 13

3.3. Breakdown of the Semiclassical Approximation . . . . . . . . . . . . . . . . . 15

3.4. Instantons in the Orientifold Model . . . . . . . . . . . . . . . . . . . . . . 17

\section{Introduction}

Recent progress in string theory has led to deep conceptual insights into the quantum nature of a number of spacetime geometries, including black holes and AdS. dS (de Sitter) has so far been largely left out of the fun. A key reason for this is that so far no fully satisfactory dS solution of string theory has been found. 1 The problem is intrinsically difficult because there can be no unbroken supersymmetry in dS [9]. Hence the solutions are likely to be isolated with no massless scalars or moduli.

A recent approach [1] employs supercritical superstring theory. Although they do not have flat space as a solution, noncritical string theories are of intrinsic interest for a wide variety of reasons. They are implicated in tachyon decay processes in compact closed string backgrounds [10], and in attempts to obtain the QCD string [11]. Their precise place in the M-theory duality web remains an outstanding question. New cosmological solutions (with a strongly coupled singularity) of supercritical string theory were discussed in [12]. The recent application to de Sitter space [1] utilizes an asymmetric orientifold construction in non-critical 12-dimensional string theory which has no moduli. The supercriticality introduces a leading-order cosmological term (dilaton potential) which aids in fixing the dilaton. By turning on RR fluxes it is possible to arrange for the dilaton to have a nontrivial minimum with a positive cosmological constant. The string coupling at the minimum is numerically, but not parametrically, small. However, as stressed in [1], the true expansion parameter about the minimum - and the nature of string perturbation theory about a

1 However there are a number of interesting constructions which may not have been fully exploited [2-8]. 
minimum which balances dilaton tadpoles from noncriticality against RR fluxes - are not understood. For both of these reasons the existence of a string perturbation expansion about the minimum is in question, and strong coupling effects could in principle eliminate the $\mathrm{dS}$ solution. A second issue in this model is that the $\mathrm{dS}$ minimum is unstable to decay to flat space. This implies that not every point on the asymptotic boundary of the space is $\mathrm{dS}$. One of the recent lessons of string theory is that the nature of the boundary can be quite important, so a theory which asymptotically decays to flat space may be very different from a "stable" dS.

In this paper we report on work in progress which improves on this construction. A generalized asymmetric orientifold construction is introduced with a new parameter: the number of dimensions $D$. By making the number of dimensions large and employing the Bousso-Polchinski mechanism [13] with the RR fluxes we are able to make the cosmological constant at the minimum parametrically small, the higher-dimensional string coupling parametrically weak, and the effective barrier to the linear dilaton regime parametrically large. Despite this improvement we have not understood the true expansion parameter about the minimum, which could therefore in principle be eliminated by strong coupling effects.

In particular, as a function of the dimensionality $D$, the number of $\mathrm{RR}$ fields is $n_{R R}=$ $2^{D}$, which dominates the spectrum at large $D$. This is potentially both a liability and an asset: on the one hand, the $2^{D} \mathrm{RR}$ species threaten to render the effective coupling uncontrollably large; on the other hand, the large number of RR fluxes facilitate the construction of vacua with small cosmological constant and weak $D$-dimensional string coupling. As one increases $D$, the naive number of degrees of freedom increases, and as we will see one can obtain a larger and larger de Sitter space. It is tempting to speculate that the $2^{D}$ RR degrees of freedom pertain to the entropy; this will be interesting to explore in the future. In particular, since a large de Sitter space requires a large number of states (to account for the large entropy), the large number of degrees of freedom intrinsic to supercritical string theory may play a natural role.

We also consider, in a more general setting, the issue of the decay of dS space to flat space. When the barrier is small such decays clearly occur via flat space bubble nucleation and are described by gravitational instantons. However, the required bubble size grows with the barrier height, and eventually the bubble wall crosses the horizon. We will argue that the inclusion of such superhorizon processes has bizarre consequences. Causality and unitarity appear to be violated, and for very large height the process describes the 
tunneling of the entire universe to a planckian region! The proper rules for dS quantum gravity are not well understood, and this casts doubt on the assertion that such instantons should be included in the first place. We further note that the tunneling time exceeds the Poincare recurrence time for $\mathrm{dS}$ [14] for exactly the same parameter range that the instanton becomes superhorizon sized. (It also exceeds the (shorter) time for all of de Sitter space to tunnel into a maximal black hole [15].) Hence both the observable significance and the validity of the semiclassical approximation are in question for the superhorizon decay processes.2 If the superhorizon instantons are excluded, a "false" dS vacuum may be stable against decay to flat space (or to the linear dilaton regime in the case of the supercritical models), or equivalently the decay time may become so long as to be meaningless.

In the supercritical models, one can in this way potentially forbid decays from a large range of dS minima to the linear dilaton regime, since as we will see the domain wall tension is too large for a sub-horizon size bubble. However, we also find decays between different flux vacua proceeding via nucleation of D-branes (as in [20,13,16]), including transitions from $\mathrm{dS}$ to $\mathrm{AdS}$. The model thus is a stringy construction sharing features with those studied in [21,20, 13, 16] exhibiting a dynamical relaxation of the cosmological constant. Among the different flux vacua, there are many more choices of flux configuration yielding larger values of the cosmological constant than smaller values, and in our system there are large degeneracies among different flux vacua due to the highly symmetric structure of the internal dimensions.

This paper is organized as follows. Section 2.1 presents the asymmetric orientifold construction. 2.2 describes the de Sitter minima, and 2.3 discusses the lower limit on the cosmological constant implied by flux quantization. 3.1 reviews the instantons which describe the tunneling from de Sitter to flat space. 3.2 questions the conventional wisdom that this tunneling occurs (or is even well-defined) for arbitrarily high barriers. 3.3 relates this to Poincare recurrence and the breakdown of the semiclassical approximation. Finally in section 3.4 we address the stability of the asymmetric orientifold models.

2 As discussed in section 3.4 and alluded to in [16], this is a de Sitter analog of the breakdown of the semiclassical approximation for black holes discussed in [17]. Related discussions can be found in 18,19. 


\section{2. de Sitter Compactifications of Super-Critical String Theory}

In this section we generalize the construction of [1] to large numbers $D$ of dimensions and describe de Sitter solutions of the low energy action. We compute the contributions to the dilaton potential from noncriticality, orientifold planes and RR fluxes. We demonstrate that by taking the number of dimensions to be large, one can find potentials having minima at a parametrically small value of the $D$-dimensional string coupling. Finally, we consider flux quantization and show that at large $D$ the cosmological constant can be made parametrically small.

\subsection{Asymmetric Orientifolds in Non-Critical String Theory}

In $\mathrm{D}$ (more than 10) dimensions, we start with the string frame low energy effective theory for the graviton, dilaton and Ramond-Ramond fields

$$
S_{D}=\frac{1}{2 \kappa_{D}^{2}} \int d^{D} x \sqrt{-G}\left(e^{-2 \phi}\left(R-\frac{2(D-10)}{3 \alpha^{\prime}}+4 \nabla_{\mu} \phi \nabla^{\mu} \phi\right)-\frac{1}{2} \sum_{p}\left(F_{p}\right)^{2}\right)
$$

where the sum runs over the various RR fields $F_{p}$ in the theory.

We will be interested in asymmetric orientifold models obtained from this $D$ dimensional theory in which the dilaton is fixed. Let us begin by noting a few salient points regarding the spectrum in these relatively unfamiliar theories. Note from the action (2.1) (and as discussed in [22] and reviewed in [四), the graviton, dilaton, and RR fields in $D$ dimensions are massless. However, if one calculates using free field theory the putative zero-point energy of these fields in flat (string-frame) space, i.e. in the linear dilaton background, one finds in the NS sector a vacuum energy of $-(D-2) / 16$. As explained in [22, this reflects the effective tachyonic behavior of the fields in the linear dilaton background (obtained from (2.1) by expanding in small fluctuations about the linear dilaton solution). In order to obtain the effective mass squared of the fields in the Lagrangian expanded around a putative extremum with constant dilaton (such as those we are studying in this paper) one must therefore cancel the contribution from the linear dilaton from the zero point energy. This amounts to the statement that in the NS sector, the effective vacuum energy $E$ is off from the free field result $E_{0}$ by

$$
E=E_{0}+\frac{D-10}{16} .
$$

Let us now proceed to the models of interest here, which are compactifications from $D$ down to $d=D-r$ dimensions. We will eventually be interested in the case of large 
$D$ with $d$ held fixed, and in particular how various quantities depend on $D$. Because as we will see the quantities relevant to our conclusions scale exponentially with $D$, some numerical factors which are order one will not be explicitly computed.

We begin with a self-dual torus $T^{r}$. The zero modes on the torus are given by

$$
\begin{aligned}
& p_{L}^{i}=\frac{1}{\sqrt{\alpha^{\prime}}}\left(m^{i}+n^{i}\right) \\
& p_{R}^{i}=\frac{1}{\sqrt{\alpha^{\prime}}}\left(m^{i}-n^{i}\right)
\end{aligned}
$$

and the dimensions of the corresponding worldsheet operators are $\left(\frac{\alpha^{\prime}}{4} p_{L}^{2}, \frac{\alpha^{\prime}}{4} p_{R}^{2}\right)$. Mod out by the orientifold group generated by

$$
\begin{gathered}
g_{1} \equiv\left(0, s^{2}\right)_{d+1} \ldots\left(0, s^{2}\right)_{d+r} \\
g_{2} \equiv(-1,1)_{d+1} \ldots(-1,1)_{d+r} \\
g_{3} \equiv \Omega I_{r} \\
g_{4} \equiv(-1)^{F}(s, s)_{d+1} \ldots(s, s)_{d+r}
\end{gathered}
$$

As in [1], we adopt the following notation. $\left(0, s^{2}\right)_{i}$ is an asymmetric shift on the $i^{\text {th }}$ coordinate, and acts as $(-1)^{n^{i}+m^{i}} .(s, s)_{i}$ is a geometric shift on the $i^{\text {th }}$ coordinate by half the circle radius, and acts as $(-1)^{m^{i}} . \Omega$ is an orientation reversal, $I_{r}$ a reflection on all $r$ coordinates of the $T^{r}$. $(-1,1)_{i}$ is a reflection on the $i^{\text {th }}$ left-moving coordinate only, and is at the heart of the moduli-fixing effect of this model, since it projects out all the untwisted NS NS moduli.

In order to check level-matching (for modular invariance) and to check for twisted moduli, we must compute the vacuum energy in all inequivalent sectors, taking into account (2.2). Let us start with the shifts. In the $\left(0, s^{2}\right)^{r}$ twisted sector, the momentum and winding lattice (2.3) is shifted so that $(m, n) \rightarrow(m+1 / 2, n+1 / 2)$, while in the $(s, s)$ sector it is shifted by $(m, n) \rightarrow(m, n+1 / 2)$. Each $\left(0, s^{2}\right)$ shift (per direction) has a rightmoving energy of $1 / 4$, while each $(s, s)$ shift (per direction) gives left and right moving energies of $1 / 16$. For the element $g_{2}=(-1,1)^{r}$, we have ground state energies

$$
\left(E_{L}=\frac{r}{8}-\frac{1}{2}, E_{R}=-\frac{1}{2}\right)
$$


This level-matches if $r=4 k$ for integer $k$. In order to avoid any massless modes (potential moduli) in this sector, we must take $k>1$. For the element $g_{2} g_{4}$ we have

$$
\left(E_{L}=\frac{r}{8}-\frac{1}{2}=\frac{k-1}{2}, E_{R}=\frac{r}{16}-\frac{1}{2}=\frac{k-2}{4}\right)
$$

requiring that $k \equiv 2 N$ be even for level-matching. As discussed in [22], in order to have a standard GSO projection, one requires $D=d+r \equiv 8 j+2$ for integer $j$. Altogether, in order to have a consistent orientifold group we need

$$
r=8 N
$$

for integer $N \geq 1$, and to have an ordinary GSO projection we need

$$
d=D-8 N=8 j+2-8 N .
$$

This model has two sets of orientifold planes - O- $(d-1)$-planes generated by the element $g_{3}$ and spacefilling O- $(D-1)$-planes generated by the T-dual element $g_{2} g_{3} g_{2}=\Omega$. We also have anti-orientifold planes, which are necessary to cancel the RR tadpoles - these are generated by the elements $g_{3} g_{4}$ and $g_{2} g_{3} g_{4} g_{2}$. The total contribution to the action due to these orientifold planes is

$$
S_{\text {Orientifold }}=\sum_{i} T_{O_{i}} \int d^{p_{i}+1} x \sqrt{-G} e^{-\phi}
$$

where $i$ runs over the orientifolds - here the orientifold group acting on the $r$ dimensions of our torus introduces $2^{r-1} O-(d-1)$ planes, $2^{r-1} \bar{O}-(d-1)$ planes, as well as the T-dual objects, an $O-(D-1)$ plane, and an $\bar{O}-(D-1)$ plane. These T-dual pairs are identified under the action of $g_{2}$, so (2.12) is just $2^{r}$ times the action for a single $O_{d-1}$ plane:

$$
\begin{aligned}
S_{\text {Orientifold }} & =2^{r} T_{O_{d-1}} \int d^{d} x \sqrt{-G} e^{-\phi} \\
& =-\frac{2^{7 / 2+D / 4} \pi^{1 / 2}}{\kappa_{D} \ell_{s}^{1-D / 2+d}} \int d^{d} x \sqrt{-G_{d}} e^{-\phi} .
\end{aligned}
$$

Here we have defined the string length

$$
\ell_{s}=2 \pi \sqrt{\alpha^{\prime}}
$$


and are using the generalized formula for the tension of an orientifold $p$-plane in $D$ dimensions derived in [1] with the assumptions listed there (which consist essentially of the procedure (2.2) for the closed-string channel modes applied to the annulus diagram),

$$
2^{D-(p+1)} T_{O_{p}}=-\frac{2^{7 / 2+D / 4} \pi^{1 / 2}}{\kappa_{D} \ell_{s}^{p+2-D / 2}} .
$$

The action of the orientifold group projects out the NS-NS moduli of the $T^{r}$, so the $d$-dimensional action for the untwisted NS-NS sector reduces to

$$
S_{\mathrm{NS}}=\frac{1}{2 \kappa_{d}^{2}} \int d^{d} x \sqrt{-G_{d}} e^{-2 \phi}\left(R_{d}-\frac{2(D-10)}{3 \alpha^{\prime}}+4 \nabla_{\mu} \phi \nabla^{\mu} \phi\right)
$$

where the $d$-dimensional gravitational coupling is

$$
\kappa_{d}^{2}=\frac{\kappa_{D}^{2}}{v \ell_{s}^{r}}=v^{-1} \ell_{s}^{d-2}
$$

$v$ here is the dimensionless effective volume of the compactification space given by

$$
\left(\int_{T^{r}} d^{4 m} x \sqrt{-G_{r}}\right)_{e f f}=v \ell_{s}^{r},
$$

and is of order one. We have taken the $D$-dimensional coupling to be $\kappa_{D}^{2}=\ell_{s}^{D-2}$. 3

Note that one could also consider multiple copies of this orientifold group acting on subtori of $T^{r}$. Each $\Omega I_{p}$ action reduces the RR spectrum by half, so this has the virtue of reducing the number of species which contribute to the effective coupling. However, there is a danger of also reducing the effective volume and thus $v$ in (2.18), thereby increasing the effective coupling. It would be interesting to determine the winner of the competition between these two effects, but for now we will stick to a single copy of the orientifold group (2.4)-(2.7).

We now turn on some RR fluxes along the compact directions (see, e.g. [23 29]). In $D$ dimensions, a $p$-form field strength wrapped on a cycle of volume $V_{p}$ will be quantized as

$$
\frac{1}{2 \kappa_{D}} \int_{V_{p}} F_{p}=\sqrt{\pi} \ell_{s}^{\frac{2 p-D}{2}} Q
$$

3 In making this choice, we are tacitly assuming that high order terms in the perturbation series will be $\leq$ order one with respect to this choice of coupling. See the discussion in [i] for more details. 
where $Q$ is an integer. Let us use a basis of cycles given by the square subtori $\subset T^{r}$. We will label these by $i=1, \ldots, 2^{r}$. Turning on RR fluxes adds a dilaton independent piece to the $d$ dimensional string frame action. Before orientifolding, there are $\left(\begin{array}{l}r \\ k\end{array}\right)$ possible $k$-form fluxes to choose from, for a total of $2^{r}$. Although some of the internal fluxes will be projected out by the orientifold action, certain flux configurations will be left invariant. These invariant combinations of fluxes from the untwisted sector of the orbifold, which involve fluxes of different rank related to each other by T-duality, will also be subject to the quantization condition inherited from the parent theory. Because our orbifold is of finite order independent of $r$, the number of invariant fluxes still scales like $2^{r}$ for large $r$ after taking into account the reduction in the RR spectrum effected by the orientifold action. Chern-Simons couplings among the many RR fields at large $D$ may also affect the spectrum in a given flux background, and the set of consistent choices of flux configuration; this would be interesting to work out in detail.

Going to $d$-dimensional Einstein frame

$$
G_{d \mu \nu} \rightarrow \tilde{G}_{\mu \nu}=G_{d \mu \nu} e^{\frac{4 \phi}{2-d}}
$$

the low energy action becomes

$$
S=\frac{1}{2 \kappa_{d}^{2}} \int d^{d} x \sqrt{-\tilde{G}}\left(\tilde{R}-\left(\frac{4}{(d-2)}\right) \partial_{\mu} \phi \partial^{\mu} \phi-\frac{1}{v \ell_{s}^{2}} U(\phi)\right) .
$$

The Einstein frame dilaton potential is

$$
U(\phi)=e^{\frac{4}{d-2} \phi}\left(a-b e^{\phi}+c e^{2 \phi}\right)
$$

where

$$
\begin{aligned}
a & =v 4 \pi^{2}\left(\frac{2(D-10)}{3}\right) \\
b & =22^{7 / 2+D / 4} \pi^{1 / 2} v_{O} \\
c & =\sum_{i=1} \frac{\pi}{v_{p_{i}}} Q_{i}^{2} \ell_{s}^{2 p_{i}-D}+\Lambda_{1} \equiv \pi \sum_{i=1} \tilde{Q}_{i}^{2}+\Lambda_{1} .
\end{aligned}
$$

Here in the expression for $b, v_{O}$ is a dimensionless volume associated with the orientifold planes on our orbifold similar to $v$; again this is of order 1 in our model and we will not keep track of such factors in our analysis. In the expression for $c, i$ labels the fluxes in the square basis discussed above, and we consider only invariant combinations of these basic fluxes. $p_{i}$ is the degree of the field strengh and $v_{p_{i}}$ is an order one dimensionless volume 
associated to the $i^{\text {th }}$ flux. (Before the orientifolding, these volumes are self-dual, but as in (2.21) the effective volumes may be reduced by the action of the orientifold group.)

$\Lambda_{1}$ is the one-loop dilaton potential. It will be proportional to $n_{R R} \sim 2^{D}$ times $\xi(D)$ where $\xi(D)$ is an unknown $D$-dependent constant, which is related to the effective loopcounting parameter in our theory. (For some insight into the scaling of loop effects in gravitational field theory as a function of dimension $D$, see [30], where factors of $1 / D$ ! appear with additional loops, providing enhanced control at large D.) Because the $2^{D}$ RR bosons dominate the spectrum, $\Lambda_{1}$ is likely to be negative in the string theoretically regulated theory, similarly to the situation in for example Scherk-Schwarz compactifications [31] and many other non-supersymmetric orbifold examples that have been analyzed in critical string theory, in which one finds the sign of $\Lambda_{1}$ to be the same as that of the difference between the number of massless fermions and bosons in the tree-level spectrum. Below, we will analyze the potential assuming conservatively that $\Lambda_{1} \sim-2^{D}$ for definiteness, but as will become clear the qualitative results apply for a large range of possible values of $\Lambda_{1}$ including those with smaller magnitude.

In principle, we should also include a renormalization of Newton's constant at the same order; this will not affect the perturbative stabilization in what follows in this section, but nonperturbatively may adjust the instanton actions in $\S 3$.

\section{2. de Sitter Solutions}

Let us write the potential as

$$
U(\phi)=\left(a-b e^{\phi}+\frac{b^{2}}{4 a}(1+\delta) e^{2 \phi}\right) e^{\frac{4 \phi}{d-2}} .
$$

There is a de Sitter solution if $U(\phi)$ has a stable minimum at positive energy. This requires that the solutions of $U^{\prime}(\phi)=0$

$$
e^{\phi_{ \pm}}=\frac{a}{d b}\left(\frac{d+2 \pm \sqrt{(d-2)^{2}-8 d \delta}}{1+\delta}\right)
$$

are real - here $\phi_{ \pm}$is the local minimum (maximum). In addition the effective cosmological constant

$$
\Lambda=U\left(\phi_{+}\right)
$$

should be greater than zero. These two conditions require that

$$
0<\delta<\frac{(d-2)^{2}}{8 d}
$$


As $\delta$ increases from the lower bound to the upper bound, $U\left(\phi_{+}\right)$increases from 0 to $\frac{a^{\frac{d+2}{d-2}} 8^{\frac{4}{d-2}}(d-2)^{2}}{b^{\frac{4}{d-2}} d(d+2)^{\frac{d+2}{d-2}}}$. and the string coupling decreases from $\frac{2 a}{b}$ to $\frac{8 a}{b(d+2)}$. Near $\delta=0$, the cosmological constant goes like

$$
U\left(\phi_{+}\right)=a\left(\frac{2 a}{b}\right)^{\frac{4}{d-2}} \delta+\mathcal{O}\left(\delta^{2}\right) .
$$

If we wish to minimize the string coupling we must take $\delta \sim \frac{(d-2)^{2}}{8 d}$. For example, in the original scenario of [⿴囗十 $(D=12, d=4)$ this gives

$$
a=\frac{8 \pi^{2}}{3}, \quad b=\pi^{1 / 2} 2^{15 / 2}, \quad \Lambda \sim \frac{1}{\left(2 \pi \alpha^{1 / 2}\right)^{4}}(0.05), \quad e^{\phi_{+}} \sim 0.11 .
$$

This has the disadvantage that $\Lambda$ is only a couple orders of magnitude above string scale. Also, the potential barrier seperating the local minimum from the global minimum at $\phi \rightarrow-\infty$ is small, so the vacuum is not very stable against tunneling effects. Let us instead try to minimize $\Lambda$ by taking $\delta \rightarrow 0$. We find that (modulo issues of flux quantization, which we will consider in the next section) we can make $\Lambda$ as small as we like, with

$$
\Lambda \sim 0, \quad e^{\phi_{+}} \sim 0.16
$$

We have found that $\Lambda$ can be made arbitrarily small, at the cost of a small increase in the string coupling. In addition, this solution is much more stable, since the potential barrier is high.

A solution with small D-dimensional string coupling is found by taking $a / b \rightarrow 0$. From the expressions (2.23) it is clear that this can always be accomplished by taking $D$ large. However, it is not clear that this implies a small true effective string coupling after compactification. The latter may for example be enhanced by the enormous multiplicity $\left(\sim 2^{D}\right)$ of RR fields. (On the other hand, if things work as in [30], there may in fact be overcompensating loop-suppression factors as a function of $D$ that preserve the smallness of the effective coupling.)

\subsection{Solutions With Small $\Lambda$}

In order to get a small cosmological constant we must take $\delta \rightarrow 0$. However, flux quantization constrains how small we can get $\delta$, and thus how small we can get $\Lambda$. We see from $\S 2.2$ that for $\Lambda \sim 0$ and large $D, c$ approaches a large value

$$
c=\pi \sum_{i} \tilde{Q}_{i}^{2}+\Lambda_{1} \rightarrow \frac{b^{2}}{4 a} \sim \frac{2^{D / 2}}{4 a}
$$


For example, this is $\frac{3072}{\pi}$ in the scenario in [1]. Since $\Lambda_{1} \sim-2^{D}$, we have

$$
\pi \sum_{i} \tilde{Q}_{i}^{2} \sim 2^{D}
$$

By taking linear combinations of many different fluxes we can tune $c$ quite accurately this is similar to the mechanism of Bousso and Polchinski [13], though in our case we have large degeneracies in the set of flux configurations. The allowed charges $Q_{i}$ lie on a $q \sim 2^{r} \sim 2^{D}$-dimensional lattice. Because of the flux quantization condition, the smallest jumps we can have in $c$ are of order 1 . Because of (2.32) and the fact that we have $2^{D}$ independent fluxes $\tilde{Q}_{i}$ to pick, there will always be some $\tilde{Q}_{i}$ which are of order 1 (or smaller), so order 1 jumps are indeed possible. Using (2.28), this gives for the scale of the lowest-lying de Sitter minima

$$
\begin{aligned}
& \Delta c \sim \frac{b^{2}}{4 a} \delta \sim 1 \\
& \Lambda=U\left(\phi_{+}\right) \sim\left(\frac{a}{b}\right)^{\frac{2 d}{d-2}} \sim 2^{-\frac{D d}{2(d-2)}}
\end{aligned}
$$

Since $b \sim 2^{D / 4}$, this vacuum energy is exponentially small for large $D$.

\section{Metastability of the de Sitter Vacuum}

In addition to the de Sitter minimum, the dilaton potential (2.22) has a global minimum with vanishing cosmological constant at $\phi \rightarrow-\infty$. Our system also has a multitude of different dS and AdS vacua obtained from different configurations of flux in the internal space. This raises the issue of whether or not the de Sitter minimum is only metastable. This question arises generically in any string construction of a de Sitter solution involving a potential which vanishes at weak coupling, and/or containing many flux vacua.

Instantons have been described [32, 20] which might be related to this tunneling. However, as we will see in this section, when the barrier between the minima is sufficiently large, the instanton degenerates and no longer describes tunneling of a de Sitter horizon volume to a comparably sized-region of flat space. The instanton describes a rather unphysical process in which the visible universe disappears altogether. Such "super-horizon" instantons occur in the parameter range for which the bubble wall lies behind the horizon.

Whether or not such processes actually occur, and whether or not such de Sitter vacua can be stable, are questions which cannot be definitively settled with our present understanding of quantum gravity in de Sitter space. In ordinary field theory, instantons provide 
saddle point approximation to a functional integral with fixed boundary conditions. The instantons which describe the decay/disappearance of de Sitter space have no boundary at all, and so it is not clear if they should be included. We will argue that the super-horizon instantons in a sense violate both causality and unitarity and should be omitted altogether. We will also discuss other potential mechanisms for mediating vacuum decay.

\subsection{The Instantons}

For simplicity we work in the thin wall approximation, in which case the relevant instanton solutions are rather simple. They have been described in detail in [20] and will now be reviewed.

The euclidean solutions are characterized by the tension $T$ of the bubble wall and the dS cosmological constant $\Lambda$. The solutions are determined by simply matching the extrinsic curvatures on the two sides of the bubble wall to the tension $T$ in accord with the Israel junction condition. The instanton looks like a portion of a round sphere glued to a portion of flat space. The spherical portion is

$$
d s^{2}=R_{d S}^{2}\left(d \theta^{2}+\sin ^{2} \theta d \Omega_{3}^{2}\right), \quad 0 \leq \theta \leq \arcsin \frac{R_{B}}{R_{d S}}
$$

where $d \Omega_{3}^{2}$ is the metric on the unit three sphere, $R_{d S}=\sqrt{3 / \Lambda}$ is the dS radius, and $R_{B}$ is the radius of the $S^{3}$ boundary. The flat space portion is

$$
d s^{2}=d r^{2}+r^{2} d \Omega_{3}^{2}, \quad 0 \leq r \leq R_{B}
$$

The full instanton is then obtained by gluing together (3.1) and (3.2) along the $S^{3}$ bubble wall at radius $R_{B}$. This is depicted in figure 1a-c. The Israel junction condition

$$
\frac{1}{R_{B}^{2}}=\frac{1}{R_{d S}^{2}}+\left(\frac{1}{T R_{d S}^{2} \kappa^{2}}-\frac{T \kappa^{2}}{4}\right)^{2}
$$

where $M_{P}$ the Planck mass, determines $R_{B}$ in terms of $T$. Note that $R_{B}$ increases with $T$ for small $T$ but then decreases for $T$ greater than the critical value

$$
T_{C}=\frac{2}{\kappa^{2} R_{d S}} .
$$


a)

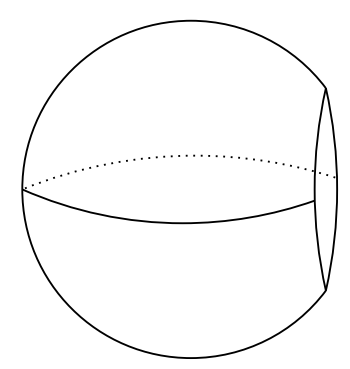

b)

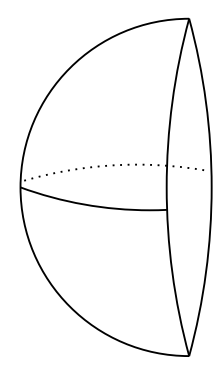

c)

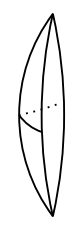

Fig. 1: The Euclidean instanton solutions matching the sphere (Euclidean de Sitter) to flat space. The cases $T<T_{C}, T=T_{C}$ and $T>T_{C}$ are shown in figures a), b) and c) respectively.

$R_{B}$ approaches zero for very large $T$.

It is straightforward to generalize these euclidean solutions to the $\mathrm{dS} \rightarrow \mathrm{dS}$ and $\mathrm{dS} \rightarrow \mathrm{AdS}$ cases. In general dimension $d$, the relation (3.3) becomes [20]

$$
\frac{1}{R_{B}^{2}}=\frac{2 \Lambda_{o}}{(d-2)(d-1)}+\left(\frac{\kappa_{d}^{2} T}{2(d-2)}+\frac{\Lambda_{i}-\Lambda_{o}}{(d-1) T \kappa_{d}^{2}}\right)^{2}
$$

Here, $\Lambda_{o}$ is the initial dS cosmological constant (outside the bubble) and $\Lambda_{i}$ is the final cosmological constant $\Lambda_{i}$ (inside the bubble). In general dimension $d$, the critical tension is

$$
T_{C}^{2}=\frac{2\left(\Lambda_{o}-\Lambda_{i}\right)(d-2)}{(d-1) \kappa_{d}^{4}} .
$$

The instanton purportedly describes tunneling from one classical geometry to another. We are interested in an initial dS geometry. The final geometry is then given by the analytic continuation of the instanton, which describes an expanding bubble of flat space inside dS. The two geometries are glued together along the moment of time symmetry. This is depicted in figure 2a-c. The tunneling rate is purportedly given by the action of the instanton minus the background action of Euclidean dS without a bubble. This is

$$
\Delta S=2 \pi^{2} R_{B}^{3} T+\frac{2 \pi^{2}}{R_{d S}^{2} \kappa^{2}}\left[2 R_{d S}^{4} \mp\left\{3 R_{d S}^{3}\left(R_{d S}^{2}-R_{B}^{2}\right)^{1 / 2}-R_{d S}\left(R_{d S}^{2}-R_{B}^{2}\right)^{3 / 2}\right\}\right] .
$$

The upper and lower signs correspond to $T<T_{C}$ and $T>T_{C}$, respectively. Again, the expression for general $d$ was worked out in [20] (equations (6.4)-(6.7)).

\subsection{Causality}

The tunneling process depicted for small tensions in figure 2a approaches the usual flat space false vacuum decay in the limit $M_{P} \rightarrow \infty$ with $T$ held fixed. The rate according 
a)

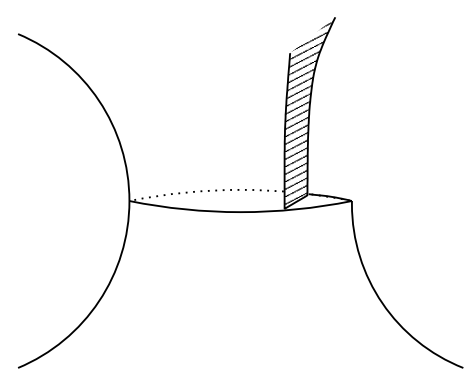

b)

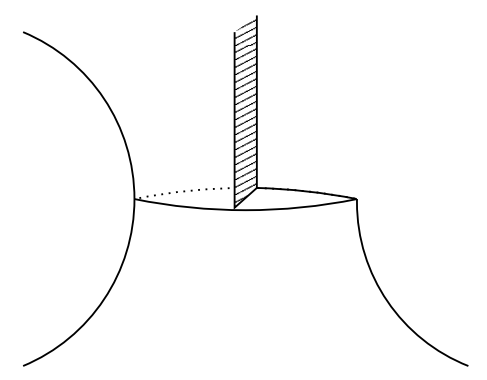

c)

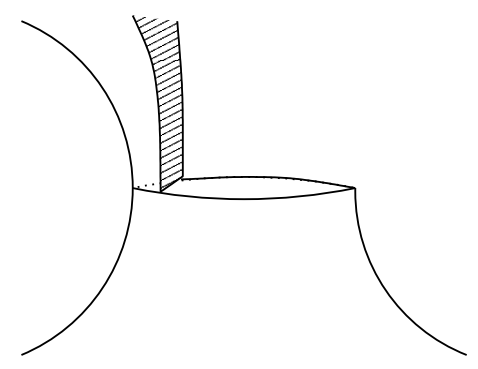

Fig. 2: The Lorentzian instanton geometry describing the nucleation of a bubble of flat space (the shaded region) inside de Sitter space. The cases $T<T_{C}, T=T_{C}$ and $T>T_{C}$ are shown in figures $\left.\left.\mathbf{a}\right), \mathbf{b}\right)$ and $\mathbf{c}$ ) respectively.

to (3.7) also approaches the correct flat space value. The instanton of figure 1a surely describes this tunneling process for sufficiently small but finite $\frac{T}{M_{P}^{3}}$.

The process depicted in figure $1 \mathrm{c}$ on the other hand has a bizarre interpretation. The entire universe tunnels to a small dime, with one flat and one dS face! Furthermore for $T \rightarrow \infty$ the rate from (3.7) approaches a constant. Hence the tunneling rate can be enhanced by adding a large number of ultra-planckian domain walls. In fact, the action (3.7) is not monotonically increasing in the regime $T>T_{C}$; for certain ranges of paramaters, the tunneling rate increases as the tension increases! This conflicts with the notion of decoupling in low-energy field theory, as well as the general fact that tunneling effects are supressed as the size of the barrier increases.

This process also appears in conflict with causality. An observer in dS should be insensitive to any physics behind the horizon. In particular there should be no consequences of placing boundary conditions on the fields along a timelike surface behind the horizon. It is easy to find boundary conditions that forbid the super-horizon instanton. Therefore the observer can learn about physics behind the horizon by waiting to see whether or not the tunneling occurs.

There is also an issue with unitarity. In the benign process of figure $2 \mathrm{a}$, an observer at the south pole finds him or herself, after the tunneling, in the middle of a bubble of flat space. However for the superhorizon case of figure $2 \mathrm{c}$, his or her entire southern causal diamond - the entire observable universe - disappears. It has been advocated by many (see for example [33] and the contribution [34] to these proceedings) that the causal diamond should be viewed as a closed unitary system (whose microstates compute the entropy). Surely this process - in which the diamond disappears altogether- violates unitarity in the worst possible manner! 
Based on these observations, our conclusion is that when the tension $T$ exceeds $T_{C}$, the superhorizon instantons simply should not be included in the semiclassical description of dS. At the same time we wish to stress that, with our current level of understanding of dS quantum gravity, no such conclusions can be drawn with certainty.

The above arguments apply equally well to tunneling from de Sitter to de Sitter or Anti-de Sitter, with the critical tension given by (3.6). We should note that the criterion

$$
T>T_{C}
$$

in the case $\Lambda_{o}=0, \Lambda_{i}<0$ reproduces the well known Coleman and DeLuccia condition for the stability of flat space against tunneling to Anti de Sitter [32]. We may thus regard the stability criterion (3.8) as a generalization of the Coleman - DeLuccia mechanism.

Even if such instantons are not to be included, there may be other processes which mediate the decay of the $\mathrm{dS}$ to flat space when the barrier is very high. For example if de Sitter space is viewed as a thermal ensemble 1 , thermal fluctuations could eventually push the value of $\phi$ over the top (see e.g. [35] [36] for a discussion of this mechanism). This however is also not obviously possible. There appears to be a maximum energy allowed in dS given by the largest black hole which can fit inside the observer horizon. If the energy required to cross the barrier to flat space exceeds this value, it may be suppressed. Furthermore if the appealing notion [37,38, 39] that dS has a finite number of states given by the area law is accepted, there must be a highest energy state. Again if this is less than the barrier height decay to flat space is suppressed.

\subsection{Breakdown of the Semiclassical Approximation}

There is yet another way to interpret the condition $T^{2}>T_{C}^{2} \sim \Lambda$, which involves a further assumption about de Sitter quantum gravity. Following [37], we assume that de Sitter gravity has a finite number of degrees of freedom which determine the de Sitter quantum entropy. Imagine in this context a detector sitting on a timelike geodesic for a very long time. The detector must be built out of a subset of the finite number of degrees of freedom, all of which will eventually be thermalized by de Sitter radiation from the horizon. This thermalization process sets a maximum timescale in de Sitter space, intervals longer than which can never be measured by a geodesic observer. (See also [18,19.) The precise

4 with temperature conjugate to the energy defined by the timelike Killing vector which preserves the causal diamond 
value of the thermalization time depends on the structure of the detector, but it is certainly less than the Poincare recurrence time, which is a timescale on which all degrees of freedom have been thermalized. This recurrence time is related to the de Sitter entropy by [14 0

$$
t_{\text {recurrence }} \sim \exp \{S\}=\exp \left\{\frac{8 \pi^{2} R_{d S}^{2}}{\kappa^{2}}\right\} .
$$

Another time scale in de Sitter is the typical time for the entire space to tunnel to a maximal sized black hole. This has been estimated using instantons in [15] as

$$
t_{\text {blackhole }} \sim\left(t_{\text {recurrence }}\right)^{1 / 3} .
$$

Hence the entire space tunnels into a maximal black hole exponentially many times before the Poincare recurrence time.

We wish to compare these times to the expected lifetime of de Sitter space due to vacuum decay. When the tension equals the critical value $T_{C}$, the lifetime for the putative instanton decay is (omitting a prefactor which is polynomial in $R_{d S}$ )

$$
t_{\text {decay }} \sim \exp \{\Delta S\}=\exp \left\{\frac{8 \pi^{2} R_{d S}^{2}}{\kappa^{2}}\right\} .
$$

This is precisely the Poincare recurrence time! Thus as $T$ approaches the critical value $T_{C}$ the lifetime becomes comparable to the recurrence time, and no observer will ever live long enough to see the vacuum decay. 6 Moreover, at $T=T_{C}$ the lifetime is much longer than the time (3.10)

$$
\begin{aligned}
t_{\text {decay }} & \sim t_{\text {recurrence }} \\
& \sim t_{\text {black hole }}^{3} .
\end{aligned}
$$

Hence in order to observe the decay of de Sitter space when $T=T_{C}$ one needs a detector capable of passing through a black hole exponentially many times. We regard the existence of such detectors doubtful!

5 The authors of [14] considered several different types of recurrence phenomena. Here we quote the timescale for two point fluctuations proportional the thermal background value of the Green function - so called "relative" fluctuations - as opposed to fluctuations of some fixed size independent of $S$.

6 Given that the action (3.7) decreases at $T>>T_{C}$ one might worry that naively applying the instanton methods for very large tensions would lead to decay timescales shorter than the recurrence time. This turns out not to be the case: as $T \rightarrow \infty$ the decay time precisely approaches (3.11). 
Let us state this in yet another way. The semiclassical approximation describes the de Sitter horizon as a hot wall in contact with a heat reservoir with infinite heat capacity. In this approximation no correlations ever appear in the radiation emitted from the horizon. In the exact theory, it is plausible that the horizon has a finite heat capacity as determined from the finite de Sitter entropy. This means that if we watch long enough correlations will be seen in the radiation. A typical time required to see those correlations is the Poincare recurrence time. Hence this time scale signals the breakdown of the semiclassical approximation. A semiclassical instanton which involves a longer time scale therefore cannot be trusted.

Phrased in this way, our argument parallels a similar one give for black holes in [17], and alluded to in the de Sitter context in [16]. In [17], it was argued that the semiclassical approximation for near-extremal black holes breaks down as the temperature goes to zero very near extremality. The breakdown occurs when the energy of a typical thermal Hawking quantum exceeds the excitation energy of the black hole above extremality. Clearly the Hawking emission cannot proceed under these circumstances because it would leave a subextremal black hole with a naked singularity.

This is a close analogy to the situation we have described in the de Sitter context. The hot horizon emits a thermal spectrum of bubbles of flat space. When the energy of these bubbles (as determined in part by the tension of the bubble walls) exceeds the energy of de Sitter space above flat space, the semiclassical approximation breaks down.

In the black hole case, it was eventually quantitatively understood [40] in the context of string theory that this breakdown of the semiclassical approximation signals the appearance of a gap. Presumably similarly interesting and yet-to-be understood phenomena appear in the de Sitter context.

In conclusion, superhorizon tunneling processes from dS to flat space do not appear to be meaningful or consistent. The stability and correct quantum description of a dS vacuum separated by a very high barrier from flat space is an open question.

7 Of course, as mentioned above, no one can live that long. However this only underscores the unphysical nature of a tunneling process which takes such a long time. 


\subsection{Instantons in the Orientifold Model}

In the asymmetric orientifold model the tension of the domain wall separating the de Sitter from the flat vacuum at $\phi \rightarrow-\infty$ is determined by the shape of $U(\phi)$; for example in $d=4$ it is roughly

$$
T \sim \frac{a^{3 / 2}}{b} .
$$

Using the criterion of the previous subsection, we conclude that many of the de Sitter

minima discussed in section 2 are stable against decay to the linear dilaton regime. 8 The maximum-energy de Sitter minimum stable under this decay is at $c \sim \frac{b^{2}}{4 a}+\mathcal{O}\left(b^{2}\right)$, i.e. at $\delta \sim 1$. (Here we are only keeping track of exponential dependence on $D$, i.e. factors of $b$ but not a.) This corresponds to an energy of the order

$$
U_{+}^{\max } \sim \frac{1}{b^{2}} \sim 2^{-D / 2}
$$

The minimum-energy de Sitter minima possible with our quantization condition on the charges and thus on $c$ (which are of course also stable under this decay) have $c \sim \frac{b^{2}}{4 a}+\mathcal{O}(1)$, i.e. at $\delta \sim \frac{a}{b^{2}}$. This corresponds to an energy of the order

$$
U_{+}^{\min } \sim \frac{1}{b^{4}} \sim 2^{-D}
$$

In addition to the instanton decays to the linear dilaton regime discussed above, there is also the possibility of transitions among the different flux vacua, as in [20] [13] [16] [41]. Dbranes extended along $d-1$ of the $d$ de Sitter dimensions constitute domain walls separating vacua with different flux configurations. More specifically, D-branes of charge $Q$ connect vacua of flux $Q_{1}$ and $Q_{1}-Q$ on the dual cycle to the D-brane on the compactification. In order to determine the (in)stability of our solutions, we must apply the results reviewed in $\S 3.1$ to such D-brane induced decays in addition to the dilatonic domain wall we considered above.

At our de Sitter minima for $d=4$, the string coupling is

$$
g_{s} \sim 1 / b
$$

8 We should note that when $D$ is large, the thin wall approximation breaks down for the potentials (2.22); in this limit the width of the domain wall interpolating between the de Sitter and flat vacua scales as $T^{-1}$. However, this subtlety does not affect the causality considerations of Section 3.2. 
and the energy is

$$
U_{+} \sim\left(a^{4} / b^{4}\right)\left(c-\left(b^{2} / 4 a\right)\right)
$$

As we just discussed, the lowest-lying dS vacua have $c$ tuned to cancel $b^{2} /(4 a)$ to within order 1 , so that

$$
U_{+}^{\min } \sim 1 / b^{4}
$$

The highest-lying dS minima that are stable against decay to the linear dilaton background have, from our earlier calculation, $c$ tuned such that $c-\left(b^{2} / 4 a\right) \sim b^{2}$, i.e.

$$
U_{+}^{\max } \sim a^{2} / b^{2}
$$

Recall from (3.6) that

$$
T_{C}^{2} \sim \Lambda_{o}-\Lambda_{i}
$$

The D-brane tension is, in Einstein frame, from [1] and the above scaling of $g_{s}$ at the minimum,

$$
T \sim\left(1 / b^{2}\right) 2^{-D / 4}
$$

This formula will apply for a transition in which the bubble wall is a single D-brane; the tension of multiple D-branes will be subject to appropriate binding energy contributions.

If we allow the instanton, i.e. if $T<T_{C}$, then its action $B$ is given by equation (6.4) in [20]. One should keep in mind that the renormalization of Newton's constant may affect the overall scaling of the action. In addition to the contribution of exp(-action) to the probability for decay, there will also be significant degeneracy factors from the large multiplicity of vacua in our large- $D$ system. Here we will confine ourselves to checking whether the transitions occur at all according to the criterion we have developed in this paper, assuming that the semiclassical instanton analysis applies (i.e. that the action is large enough in renormalized Planck units).

For example, consider decays from $U_{+}^{\max } \rightarrow U_{+}^{\max }-a^{4} / b^{4}$. This occurs if $c \sim \sum\left(\tilde{Q}_{i}\right)^{2}$ changes by order 1 , and in particular can proceed via a bubble consisting of a single D-brane . In this case, the D-brane tension is

$$
T_{(i)} \sim 2^{-D / 4}\left(1 / b^{2}\right)
$$

while the critical tension in this case is

$$
T_{C(i)} \sim 1 / b^{2}
$$


So $T_{(i)}<<T_{C(i)}$, and the decay proceeds according to our criterion developed above.

Similarly, there are decays from dS to AdS. Consider for example a transition $U_{+}^{\text {min }} \rightarrow$ $-U_{+}^{m i n}$. Here again

$$
T_{(i i i)} \sim 2^{-D / 4} 1 / b^{2}
$$

and

$$
T_{C(i i i)} \sim 1 / b^{2}
$$

so the decay is again allowed.

As we mentioned above, there will be large factors in the transition rates associated with the relative multiplicity of different decay endpoints. In particular, the smaller the value of $\sum \tilde{Q}_{i}^{2} \equiv R^{2}$ coming into the coefficient $c$, the fewer choices of flux configuration there are in the window between $R$ and $R+\Delta R$ for a fixed $\Delta R$. So although decays to AdS are possible, it is reassuring that this degeneracy factor prefers the less negative $\Lambda_{i}$ values. (In fact these factors also prefer higher $\mathrm{dS}$ vacua to lower ones, which may act to suppress the decays depending on the scaling of the renormalized instanton action.)

\section{Acknowledgements}

We are grateful to M. Aganagic, T. Banks, R. Bousso, S. Kachru, A. Karch, A. Linde, S. Minwalla, L. Motl, L. Susskind, N. Toumbas and A. Vilenkin for useful conversations. This work was supported in part by DOE grant DE-FG02-91ER40654 and under contract DE-AC03-76SF00515 and by the A.P. Sloan Foundation. 


\section{References}

[1] E. Silverstein, "(A)dS backgrounds from asymmetric orientifolds," arXiv:hep-th/0106209.

[2] S. J. Gates and B. Zwiebach, "Gauged N=4 Supergravity Theory With A New Scalar Potential," Phys. Lett. B 123, 200 (1983).

[3] A. Chamblin and N. D. Lambert, "de Sitter space from M-theory," Phys. Lett. B 508, 369 (2001) [arXiv:hep-th/0102159].

[4] R. Kallosh, "N = 2 supersymmetry and de Sitter space," arXiv:hep-th/0109168.

[5] G. W. Gibbons and C. M. Hull, "de Sitter space from warped supergravity solutions," arXiv:hep-th/0111072.

[6] C. M. Hull, "de Sitter space in supergravity and M theory," JHEP 0111, 012 (2001) [arXiv:hep-th/0109213].

[7] P. Berglund, T. Hubsch and D. Minic, "de Sitter spacetimes from warped compactifications of IIB string theory," arXiv:hep-th/0112079.

[8] P. Fre, M. Trigiante and A. Van Proeyen, "Stable de Sitter Vacua from N=2 Supergravity," arXiv:hep-th/0205119.

[9] K. Pilch, P. van Nieuwenhuizen and M. F. Sohnius, "De Sitter Superalgebras And Supergravity," Commun. Math. Phys. 98, 105 (1985).

[10] S. P. de Alwis, J. Polchinski and R. Schimmrigk, "Heterotic Strings With Tree Level Cosmological Constant," Phys. Lett. B 218, 449 (1989).

[11] A. M. Polyakov, "The wall of the cave," Int. J. Mod. Phys. A 14, 645 (1999) [arXiv:hep-th/9809057].

[12] B. Craps, D. Kutasov and G. Rajesh, "String Propagation in the Presence of Cosmological Singularities," arXiv:hep-th/0205101.

[13] R. Bousso and J. Polchinski, "Quantization of four-form fluxes and dynamical neutralization of the cosmological constant,"JHEP 0006, 006 (2000)[arXiv:hep-th/0004134.

[14] L. Dyson, J. Lindesay and L. Susskind, "Is there really a de Sitter/CFT duality," arXiv:hepth/0202163.

[15] P. Ginsparg and M. J. Perry, "Semiclassical Perdurance Of De Sitter Space," Nucl. Phys. B 222, 245 (1983).

[16] J. L. Feng, J. March-Russell, S. Sethi and F. Wilczek, "Saltatory relaxation of the cosmological constant," Nucl. Phys. B 602, 307 (2001) [arXiv:hep-th/0005276].

[17] J. Preskill, P. Schwarz, A. D. Shapere, S. Trivedi and F. Wilczek, "Limitations on the statistical description of black holes," Mod. Phys. Lett. A 6, 2353 (1991).

[18] R. Bousso, "Adventures in de Sitter space", hep-th/0205177.

[19] R. Bousso, O. DeWolfe and R. C. Myers, "Unbounded entropy in spacetimes with positive cosmological constant," arXiv:hep-th/0205080, .

[20] J. D. Brown and C. Teitelboim, "Neutralization Of The Cosmological Constant By Membrane Creation,"Nucl. Phys. B 297, 787 (1988). 
[21] L. F. Abbott, "A Mechanism For Reducing The Value Of The Cosmological Constant,"Phys. Lett. B 150, 427 (1985).

[22] A. H. Chamseddine, "A Study of noncritical strings in arbitrary dimensions," Nucl. Phys. B 368, 98 (1992).

[23] K. Dasgupta, G. Rajesh and S. Sethi, "M theory, orientifolds and G-flux," JHEP 9908, 023 (1999) [arXiv:hep-th/9908088.

[24] S. Kachru, M. Schulz and S. Trivedi, "Moduli stabilization from fluxes in a simple IIB orientifold," arXiv:hep-th/0201028.

[25] A. R. Frey and J. Polchinski, "N = 3 warped compactifications," arXiv:hepth/0201029.

[26] S. Gukov, C. Vafa and E. Witten, "CFT's from Calabi-Yau four-folds," Nucl. Phys. B 584, 69 (2000) [Erratum-ibid. B 608, 477 (2001)] [arXiv:hep-th/9906070.

[27] S. B. Giddings, S. Kachru and J. Polchinski, "Hierarchies from fluxes in string compactifications," arXiv:hep-th/0105097.

[28] K. Becker and M. Becker, "M-Theory on Eight-Manifolds," Nucl. Phys. B 477, 155 (1996) [arXiv:hep-th/9605053].

[29] J. Polchinski and A. Strominger, "New Vacua for Type II String Theory," Phys. Lett. B 388, 736 (1996) [arXiv:hep-th/9510227].

[30] A. Strominger, "The Inverse Dimensional Expansion In Quantum Gravity," Phys. Rev. D 24, 3082 (1981).

[31] R. Rohm, "Spontaneous Supersymmetry Breaking In Supersymmetric String Theories," Nucl. Phys. B 237, 553 (1984).

[32] S. R. Coleman and F. De Luccia, "Gravitational Effects On And Of Vacuum Decay," Phys. Rev. D 21, 3305 (1980).

[33] J. M. Maldacena and A. Strominger, "Statistical entropy of de Sitter space," JHEP 9802, 014 (1998) [arXiv: gr-qc/9801096].

[34] L. Susskind, "Twenty years of debate with Stephen," arXiv:hep-th/0204027.

[35] A. D. Linde, "Hard art of the universe creation (stochastic approach to tunneling and baby universe formation)," Nucl. Phys. B 372, 421 (1992) [arXiv:hep-th/9110037].

[36] J. Garriga and A. Vilenkin, "Recycling universe," Phys. Rev. D 57, 2230 (1998) [arXiv:astro-ph/9707292].

[37] T. Banks, "Cosmological Breaking Of Supersymmetry?," Int. J. Mod. Phys. A 16, 910 (2001).

[38] R. Bousso, "Positive vacuum energy and the N-bound," JHEP 0011, 038 (2000) [arXiv:hep-th/0010252].

[39] W. Fischler, unpublished.

[40] J. M. Maldacena and L. Susskind, "D-branes and Fat Black Holes," Nucl. Phys. B 475, 679 (1996) [arXiv:hep-th/9604042].

[41] S. Kachru, J. Pearson and H. Verlinde, "Brane/flux annihilation and the string dual of a non-supersymmetric field theory," arXiv:hep-th/0112197. 